\title{
Binocular interaction: contrast matching and contrast discrimination are predicted by the same model
}

\author{
DANIEL H. BAKER *, TIM S. MEESE and MARK A. GEORGESON \\ School of Life and Health Sciences, Aston University, Birmingham, B4 7ET, UK
}

Received 30 June 2006; accepted 10 October 2006

\begin{abstract}
How do signals from the 2 eyes combine and interact? Our recent work has challenged earlier schemes in which monocular contrast signals are subject to square-law transduction followed by summation across eyes and binocular gain control. Much more successful was a new 'two-stage' model in which the initial transducer was almost linear and contrast gain control occurred both preand post-binocular summation. Here we extend that work by: (i) exploring the two-dimensional stimulus space (defined by left- and right-eye contrasts) more thoroughly, and (ii) performing contrast discrimination and contrast matching tasks for the same stimuli. Twenty-five base-stimuli made from $1 \mathrm{c} / \mathrm{deg}$ patches of horizontal grating, were defined by the factorial combination of 5 contrasts for the left eye $(0.3-32 \%)$ with five contrasts for the right eye $(0.3-32 \%)$. Other than in contrast, the gratings in the two eyes were identical. In a 2IFC discrimination task, the base-stimuli were masks (pedestals), where the contrast increment was presented to one eye only. In a matching task, the basestimuli were standards to which observers matched the contrast of either a monocular or binocular test grating. In the model, discrimination depends on the local gradient of the observer's internal contrastresponse function, while matching equates the magnitude (rather than gradient) of response to the test and standard. With all model parameters fixed by previous work, the two-stage model successfully predicted both the discrimination and the matching data and was much more successful than linear or quadratic binocular summation models. These results show that performance measures and perception (contrast discrimination and contrast matching) can be understood in the same theoretical framework for binocular contrast vision.
\end{abstract}

Keywords: Human vision; interocular suppression; masking; binocular vision; contrast gain control.

\section{INTRODUCTION}

How signals from the two eyes combine and interact has received a resurgence of interest in neurophysiology (Li et al., 2005; Macknik and Martinez-Conde, 2004;

\footnotetext{
*To whom correspondence should be addressed. E-mail: bakerdh@aston.ac.uk This paper was presented at the annual meeting of the Applied Vision Association, Bradford UK (2006).
} 
Sengpiel and Vorobyov, 2005; Truchard et al., 2000; Walker et al., 1998) and in psychophysics (Ding and Sperling, 2006; Georgeson et al., 2005; Maehara and Goryo, 2005; Meese and Hess, 2004, 2005; Meese et al., 2004, 2006; Petrov and Mckee, 2006). Our recent work on contrast masking has challenged a longstanding psychophysical framework in which the nonlinear contrast responses of left and right eyes are summed prior to contrast gain control (Legge, 1984b; Meese and Hess, 2004). Instead, we proposed a new, two-stage model (Fig. 1), in which contrast gain control occurs both before and after binocular summation (Meese et al., 2006). In a key experiment, contrast discrimination for sine-wave gratings was measured at ten pedestal contrast levels, where pedestal and test were presented to: (a) one eye only (monocular), (b) different eyes (dichoptic) and (c) both eyes (binocular). The new model correctly described: (i) binocular summation at and around threshold that was markedly greater than $\sqrt{2}$, (ii) slight facilitation in the dichoptic condition, and (iii) a complex pattern of psychometric slopes (Weibull $\beta$ ) across all three conditions (Meese et al., 2006). Campbell and Green's (1965) threshold model, and our implementation of Legge's (1984b) suprathreshold model were unable to account for any of these features.

\section{Two-stage model of contrast gain control}

Figure 1 is a schematic illustration of the two-stage model. It receives input from corresponding retinal points in the left and right eyes in the same orientation and spatial frequency bands. At the initial stage of contrast gain control (stage 1 in Fig. 1) suppression occurs both within and between the eyes. Formally, the output

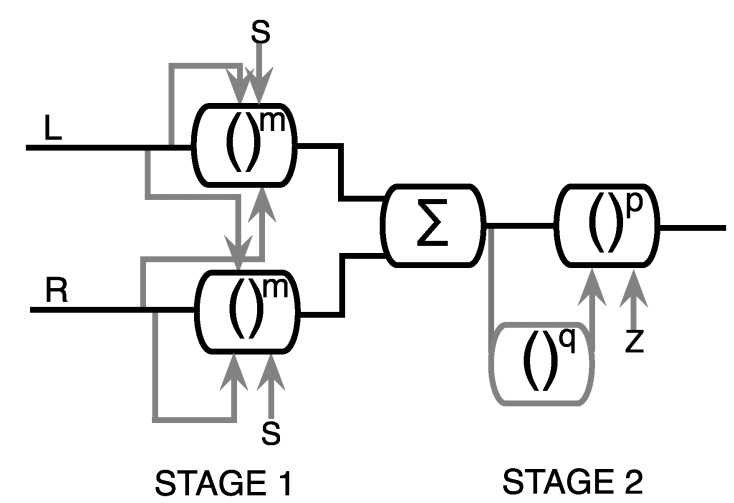

Figure 1. Schematic diagram of the two-stage model of contrast gain control (Meese et al., 2006). $L$ and $R$ are the grating contrasts in the left and right eye respectively. The black and grey lines indicate excitatory and suppressive pathways, respectively. Brackets raised to a power denote exponentiation, arrows denote division, and $\sum$ denotes linear binocular summation. Other symbols are model parameters as described in the text. Not shown is late additive Gaussian noise on the output of the model. 
of the binocular summation stage (binsum) is given by:

$$
\text { binsum }=\frac{L^{m}}{S+L+R}+\frac{R^{m}}{S+R+L},
$$

where $L$ and $R$ denote the left and right eye contrasts respectively (in \%), $m$ is the exponent of the initial accelerating nonlinearity and $S$ is the saturation constant for the first stage of gain control.

For monocular or binocular stimulation at contrast $C$, the response (before or after binocular summation) is approximately proportional to $C^{m}$ at very low contrasts, and $C^{m-1}$ at higher contrasts. Typically, we have found that $m \sim 1.3$ (Georgeson and Meese, 2005; Meese et al., 2006). To a first approximation, the binocular summation ratio (the ratio of binocular to monocular contrast sensitivities at threshold) is given by $2^{1 / m} \sim 1.7$ (Baker et al., 2006; Georgeson and Meese, 2005; Meese and Hess, 2004, 2005; Meese et al., 2006). To account for the magnitude of facilitation produced by low contrast pedestals, the final model output is subject to a second stage of nonlinear gain control (stage 2 in Fig. 1) given by:

$$
\text { resp }=\frac{\text { binsum }^{p}}{Z+\text { binsum }^{q}},
$$

where the exponents $p$ and $q$, and the saturation constant $Z$ are free parameters. In previous work we have found that $p$ is quite large (about 6 or 7). Furthermore, if $p>(q+1)$, as it can be (Meese et al., 2006), then the second stage imposes expansion (not compression) on the signal. Exactly how the second stage should be interpreted is presently unclear, as several components might contribute to its form including: static nonlinearities (Legge and Foley, 1980), dynamic contrast gain control (Foley, 1994; Heeger, 1992), stimulus uncertainty (McIlhagga, 2004; Pelli, 1985; Petrov et al., 2006), multiplicative noise (Kontsevich et al., 2002; McIlhagga and Peterson, 2006) and local light adaptation (Kingdom and Whittle, 1996; McIlhagga and Peterson, 2006). At present, we treat it as a mathematical convenience.

A sixth model parameter, $k$, is the incremental response needed to achieve a prescribed level of performance $\left(d^{\prime}\right)$. For a given $d^{\prime}, k$ is proportional to the (fixed) standard deviation $\sigma$ of late additive noise. Thus at threshold:

$$
r e s p_{\text {mask+test }}-r e s p_{\text {mask }}=\sigma \cdot d^{\prime}=k \text {. }
$$

This model provided an excellent account of the pedestal masking results described at the beginning of the Introduction. But a good challenge for any model is to test it on a stimulus set other than that for which it was developed. The stimulus conditions used by Meese et al. (2006) sampled only parts of the available stimulus space. This is shown in Fig. 2, where binocular model responses are plotted as a twodimensional function of the contrasts presented to each eye on linear (Fig. 2(a)) and logarithmic (Fig. 2(b)) axes. The height of the surface at a given point represents the magnitude of the binocular response, here scaled in units of $\sigma$. Performance 
in a contrast discrimination task requires adding sufficient contrast in one or both eyes to increase the response by around one unit from that produced by the mask (depending upon the percent correct chosen for threshold). Examples of responses to various masks are shown by the solid symbols in Fig. 2(b). Discrimination thresholds depend on the gradient of the model surface on linear axes (Fig. 2(a)). At these points, steep and shallow gradients predict low and high discrimination thresholds, respectively. The direction in which the gradient should be considered depends on the stimulus condition as follows. In the case of monoptic masking, the contrast increment is made to the one eye that also sees the mask. Thus the relevant gradient for a monoptic grating presented to the right eye is shown by the curve to the right of Fig. 2(b). In the binocular case, both eyes see the mask and the test, and so the relevant gradient direction is that which bisects the two axes (centre of Fig. 2(b)). For dichoptic masking, the mask is in one eye (left in Fig. 2) and the test is in the other (right in Fig. 2). Therefore, the relevant model gradient is at right angles to the mask contrast axis (left-hand side of Fig. 2(b)). In this case, the lengths of the gradient vectors provide a clear visual indication of the contrast
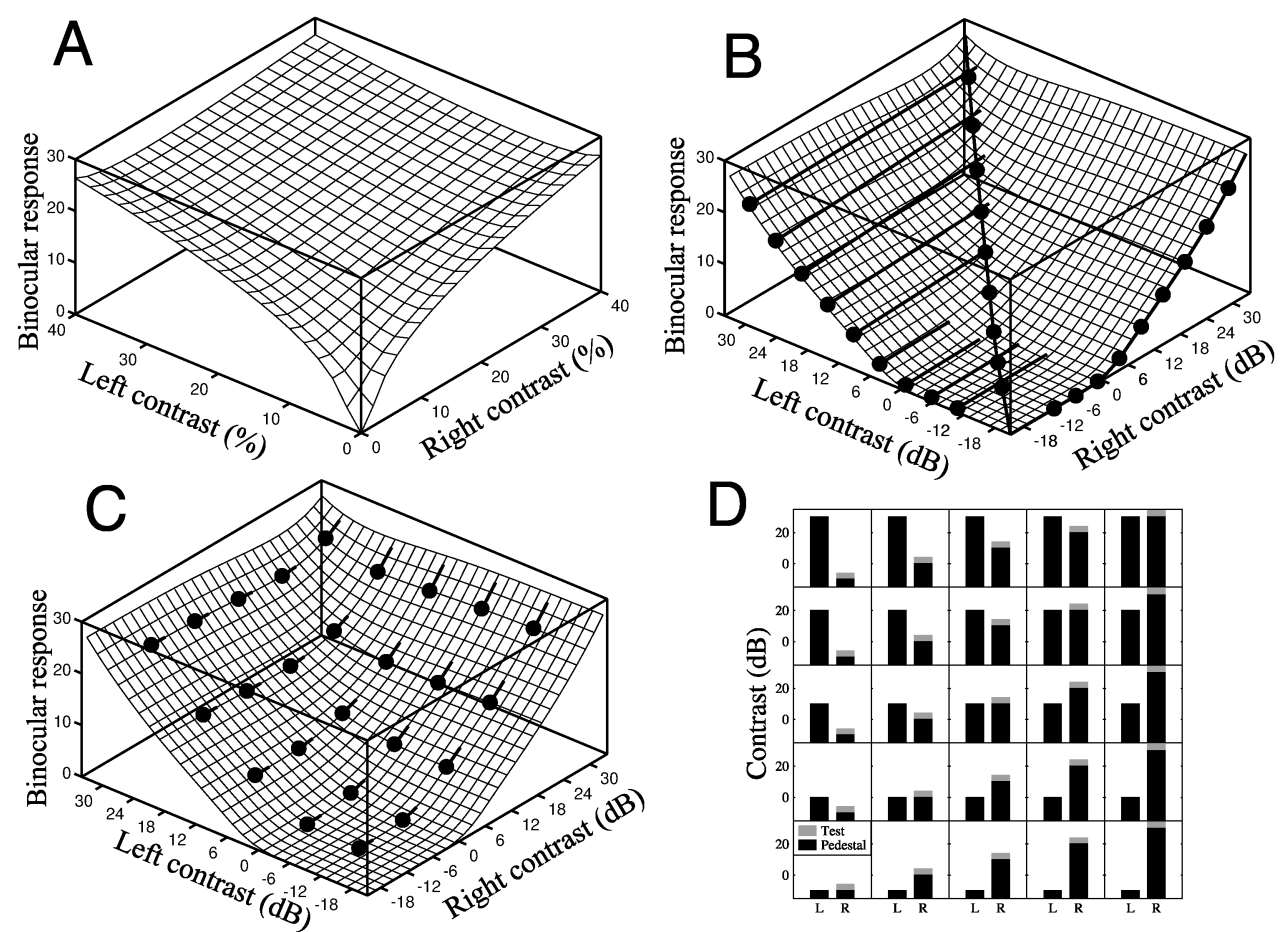

Figure 2. Binocular model responses plotted on (a) linear and (b), (c) logarithmic contrast axes. Lines and symbols in (b) refer to the three stimulus conditions investigated by Meese et al. (2006). From left to right they are: dichoptic, binocular and monocular masking. The stimuli used in the experiments here are summarised in (c), (d). These consist of independent contrast levels for left- and right-eye pedestals and a monocular test increment. In the experiments the stimuli were counterbalanced across eye. See text for further details. 
discrimination threshold for each dichoptic mask contrast. This helps to illustrate why dichoptic masking is so severe: the model response surface is fairly steep for gradient directions radiating out from the origin, but is very shallow at right angles to these.

If the response surface of Fig. 2 is a good description of binocular vision in general, then it should provide good predictions for masks and gradient directions other than those investigated by Meese et al. (2006), shown in Fig. 2(b). In Experiment I, we generated masks that sampled the stimulus space in a $5 \times 5$ matrix shown by the solid symbols in Fig. 2(c). The test increment was always in one eye only, and is shown for the right eye in Fig. 2(c), though in the experiments we counterbalanced across eye. (Note that in Fig. 2(c), the vectors indicate the direction in which the response gradient is to be assessed; their lengths do not indicate the sizes of the discrimination thresholds.) Figure 2(d) depicts the condition matrix in a different form. The black bars show pedestal contrasts for each eye, and the grey regions indicate test increments.

The model used to produce the response surfaces in Fig. 2 was derived entirely from contrast discrimination data (Meese et al., 2006). However, we wondered whether it might also provide the basis for perception of signal strength. In this case, any two stimuli that produce points at the same height on the model surface should appear to have the same contrast (Legge and Rubin, 1981). We tested this in Experiment II, where we matched the perceived contrast of monocular and binocular gratings to the same set of twenty-five stimuli used as masks in Experiment I.

\section{METHODS}

\section{Apparatus}

Presentation of independent contrasts to each eye was achieved using a frame interleaving technique, in conjunction with Ferro-Electric shutter goggles (Cambridge Research Systems, Ltd., UK) and a fast-phosphor monitor (Clinton Monoray) running at $120 \mathrm{~Hz}$. Extensive calibration confirmed there was no appreciable crosstalk between the eyes with this setup. The output of the display was linearized using standard gamma correction techniques, and had an effective luminance of $20 \mathrm{~cd} / \mathrm{m}^{2}$ when viewed through the goggles, which act as a neutral density filter. Stimulus presentation was controlled by a PC using either a ViSaGe (Experiment I) or VSG 2/5 (Experiment II) framestore (Cambridge Research Systems, Ltd., UK) operating in pseudo 15 bit mode.

\section{Stimuli}

In both experiments, the test stimulus was a horizontal 1 cycle per degree sinusoidal grating, displayed in a circular aperture $5 \mathrm{deg}$ in diameter and blurred at each edge by a 1 deg sine-wave ramp. On each trial, stimuli were presented at a phase selected 
randomly from four possibilities $\left(0,90,180\right.$ or $\left.270^{\circ}\right)$ relative to a central fixation point. The phase was the same in both intervals of a trial.

Contrast is expressed in decibels $(\mathrm{dB})$, defined as $20 \cdot \log _{10}(C \%)$, where $C \%$ is Michelson contrast in percent. Contrast pairs for left and right eyes were determined by factorial combination of 5 contrast levels $(-10,0,10,20,30 \mathrm{~dB})$. This gave 25 pairs, which were used as masks in the discrimination task (Experiment I) and standards in the matching task (Experiment II). The stimulus pair at $[-10-10] \mathrm{dB}$ was omitted from Experiment II as it was below threshold and inappropriate for a matching task.

\section{Observers and order of experiments}

The same two subjects took part in both experiments. DHB (author, male, age 23) and DJH (male, age 30) were both experienced psychophysical observers and were emmetropic, with no abnormalities of binocular or stereo vision. Both observers had also served in Experiment II of Meese et al. (2006) approximately 4 months before Experiment I here. Their results for that experiment were used to constrain the model here (see below).

\section{Procedure}

A two-interval forced-choice (2IFC) design was used. Test contrast levels were determined by an adaptive staircase routine, using either a 1-up, 3-down rule (Experiment I), or a 1-up 1-down rule (Experiment II), and terminating after 70 trials or 12 reversals, whichever occurred first. In Experiment I, the staircase determined the contrast increment in the test interval, applied to one eye only, and had a minimum step size of $3 \mathrm{~dB}$. In Experiment II, the staircase controlled the contrast level of the matching stimulus, which was either monocular or binocular, and had a minimum step size of $1.5 \mathrm{~dB}$. For monocular stimuli, the other eye viewed a zerocontrast display at mean luminance.

Subjects were seated in a dark room, $114 \mathrm{~cm}$ from the display, with their head in a support to which the goggles were attached. Responses were given using the left and right buttons of a mouse. In Experiment I, auditory feedback was given for correct or incorrect responses. No feedback was given for the subjective task in Experiment II.

Stimuli were blocked by mask or standard contrast, and run in sessions of five randomly chosen blocks (as one condition was omitted in Experiment II, there was one session of four blocks). Two staircases were interleaved within each block to counterbalance stimulus conditions across eyes. In Experiment II, the monocular and binocular matching conditions were also interleaved. Subjects were permitted to rest between blocks and at the end of sessions, which typically lasted between 10 and $20 \mathrm{~min}$. Each subject completed six repetitions of Experiment I, and either four (DHB) or one (DJH) repetition of Experiment II. 
Data were collapsed across repetition and eye, and analyzed separately for each subject using probit analysis (Finney, 1971). In Experiment I, threshold was the $75 \%$ correct point on the fitted psychometric function, based on around 600 trials for each estimate. In Experiment II, the point of subjective equality (PSE) between the standard and test contrasts was the 50\% point on the psychometric function. Reliable estimates of the PSE require considerably fewer trials than discrimination thresholds, and were based on around 160 trials for DHB and 40 trials for DJH. The reliability of these estimates was confirmed by small standard errors (determined by probit analysis), and very high repeatability across sessions for DHB.

\section{RESULTS (EXPERIMENT I): CONTRAST DISCRIMINATION}

The results from the discrimination experiment (Experiment I) are shown in Fig. 3 and are similar in form for both subjects. Each curve can be thought of as a monocular pedestal masking function (abscissa) in the presence of a dichoptic mask component whose contrast is indicated by the different symbols. For low contrast dichoptic masks (open circles and upwards triangles), the masking functions are similar to standard monocular dipper-functions (Legge, 1984a), with a region of facilitation at low pedestal contrasts and a power-law region of masking at higher pedestal contrasts. As the dichoptic mask contrast increases (other symbols), the dipper region of the function is preserved, but shifts upwards and to the right. In fact, not only does facilitation remain intact, but the dip is deeper at the highest dichoptic mask contrasts. For example, when the mask is $30 \mathrm{~dB}(32 \%)$ (solid squares), facilitation reaches as much as $18 \mathrm{~dB}$ (a factor of eight), whereas at the

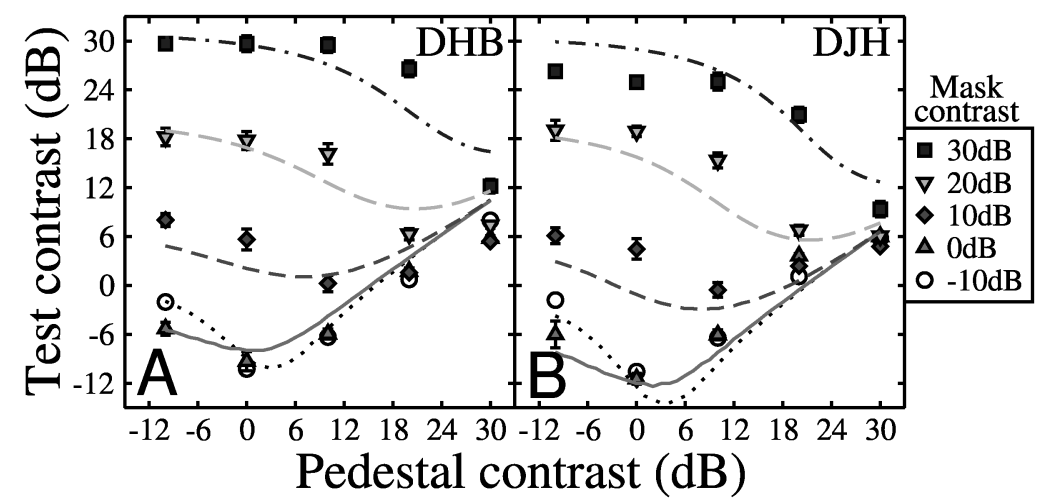

Figure 3. Discrimination thresholds and model predictions for Experiment I. The abscissa indicates pedestal contrast (in the same eye as the test), different symbols indicate the mask contrast (in the eye contralateral to the test), and the ordinate indicates the discrimination thresholds. Error bars show $\pm 1 \mathrm{SE}$ of the probit fit used in calculating the discrimination thresholds. Curves are model predictions, for which parameter values are shown in the top two rows of Table 1. The RMS errors were $2.82 \mathrm{~dB}$ and $2.75 \mathrm{~dB}$ for DHB and DJH respectively. 
Table 1.

Model parameters and RMS errors for predictions and fits of the two-stage model

\begin{tabular}{|c|c|c|c|c|c|c|c|c|c|}
\hline \multirow{2}{*}{$\begin{array}{l}\text { Data } \\
\text { source for } \\
\text { setting } \\
\text { model } \\
\text { parameters }\end{array}$} & \multirow[t]{2}{*}{ Observer } & \multirow{2}{*}{$\begin{array}{l}\text { RMS error } \\
\text { (dB) of fit }\end{array}$} & \multirow{2}{*}{$\begin{array}{l}\text { RMS error }(\mathrm{dB}) \\
\text { for predictions } \\
\text { (no free } \\
\text { parameters) for } \\
\text { Experiment I }\end{array}$} & \multicolumn{6}{|c|}{ Two-stage model parameters } \\
\hline & & & & $m$ & $S$ & $p$ & $q$ & $Z$ & $k$ \\
\hline \multirow[t]{2}{*}{ Meese et al. (2006) } & DHB & 1.39 & 2.82 & 1.19 & 0.65 & 8.61 & 6.75 & 0.16 & 0.14 \\
\hline & DJH & 1.73 & 2.75 & 1.28 & 0.89 & 6.45 & 5.15 & 0.19 & 0.09 \\
\hline \multirow[t]{2}{*}{ Experiment I } & DHB & 1.54 & - & 1.50 & 0.20 & 7.50 & 6.57 & 1.29 & 0.16 \\
\hline & DJH & 1.77 & - & 1.44 & 0.60 & 8.18 & 7.07 & 0.21 & 0.16 \\
\hline
\end{tabular}

The top two rows are for parameters sets derived from fits to the contrast discrimination data of Meese et al. (2006). The RMS errors for the predictions for Experiment I (Fig. 3) are also shown. The bottom two rows are for a direct fit to the results of Experiment I. (Numbers in bold highlight the RMS errors for the data from Experiment I.)

lowest mask contrast of $-10 \mathrm{~dB}(0.32 \%)$, the dipper has a depth of only $9 \mathrm{~dB}$ (a factor of 2.8).

To set the six parameters in the two-stage model, the thresholds from Experiment II of Meese et al. (2006) (described above) were recalculated for the two observers here using the same methods as in the present work. The best fitting parameters for each subject were then determined using a downhill simplex algorithm (Nelder and Mead, 1965). The two-stage model (Fig. 1) parameters are summarized in Table 1 and the curves in Fig. 3 show the model predictions (no free parameters) for the contrast discrimination experiment here. The model describes the general form of the new data very well, including the lateral shift of the dip and the modulation of its depth by mask contrast.

The persistence of facilitation in the presence of a fixed contrast mask is analogous to that found in other pedestal plus mask experiments (Foley, 1994; Holmes and Meese, 2004; Mullen and Losada, 1994; Ross and Speed, 1991; Ross et al., 1993), where the mask and pedestal had different image characteristics (e.g. different orientations, spatial frequencies, chromaticities). The translation of the dip in those experiments has been taken as evidence that the mask and pedestal were processed by different visual mechanisms (e.g. Holmes and Meese, 2004; Mullen and Losada, 1994). The data and modelling here are consistent with the idea that the initial processing stages for the pedestal and mask are different. However, it is clear that the translation of the dip can survive a subsequent convergence of the pathways, even when further transformations in the common pathway are involved in controlling visual performance (in the present case, this involves the parameters $p, q, Z$ and $k$ ). In particular (and as we outlined in the Introduction), the exponent $p$ is responsible for the most substantial part of the accelerating nonlinearity in the excitatory pathway (and therefore the dip), even though it is placed in the common binocular pathway. 
For completeness, we also fitted the two-stage model directly to the present data set (six free parameters; fit not shown). This improved the goodness of fit, reducing the RMS error by $1.28 \mathrm{~dB}$ for DHB and $0.98 \mathrm{~dB}$ for DJH. The RMS errors of these fits were comparable to those achieved in the fitting to the data from Meese et al. (2006) (see Table 1 for details).

\section{RESULTS (EXPERIMENT II): CONTRAST-MATCHING}

The results of the matching experiment (Experiment II) are shown in Fig. 4. Upper panels are for matches to a monocular test (test contrast in one eye only) and lower panels are for matches to a binocular test (same test contrast in both eyes). For ease of exposition, we have used nominal 'right' eye and 'left' eye labels in Fig. 4 (comparable to the 'mask' and 'pedestal' labels used in Fig. 3, respectively), but in the experiments, the stimuli were counterbalanced across eye. Curves are model predictions obtained using the parameters from the Meese et al. (2006) data set, as

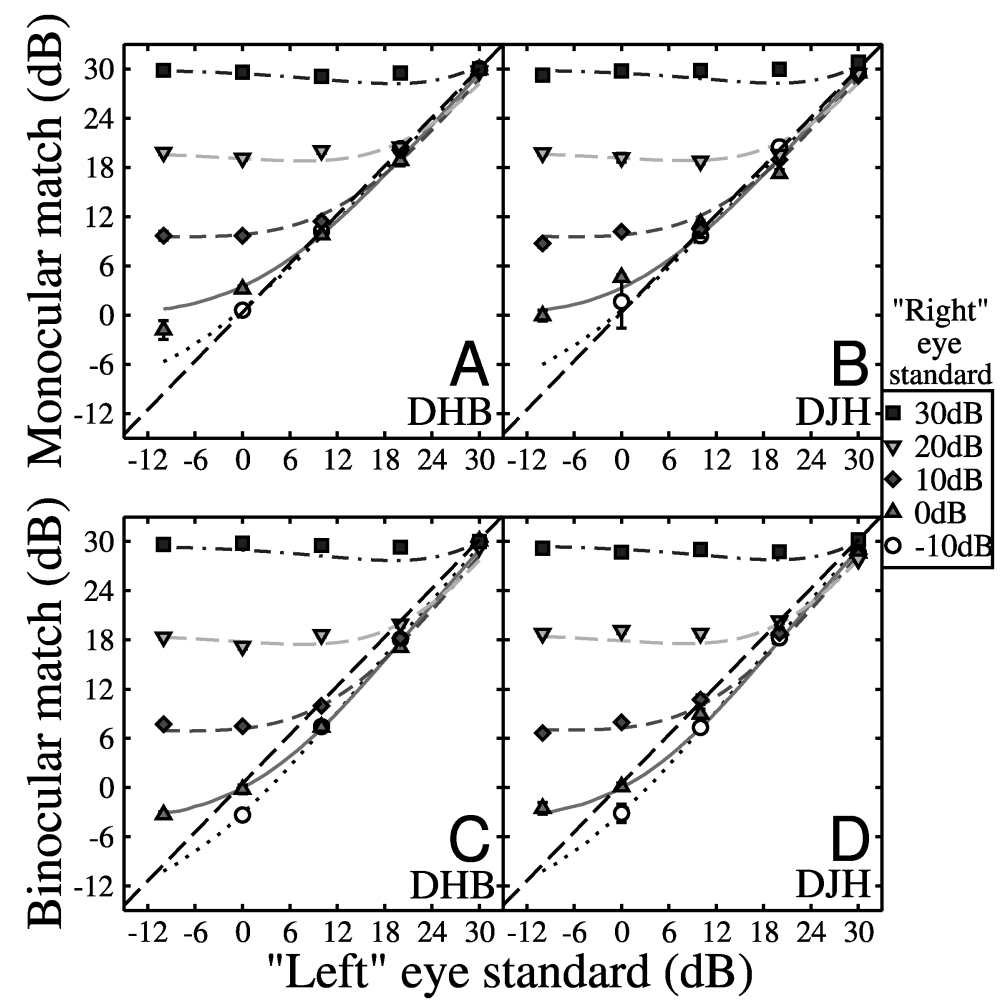

Figure 4. Contrast-matching functions for matching test gratings (ordinate) to standard gratings, for which different levels of contrast were presented to each eye (abscissa and legend). (a), (b) monocular test grating; (c), (d) binocular test grating. Error bars show $\pm 1 \mathrm{SE}$ of the probit fit, mostly smaller than symbol size. Curves are predictions of the two-stage model (Meese et al. (2006) parameters; see Table 1). 
Table 2.

RMS errors for three different models (no free parameters) for Experiment II

\begin{tabular}{|c|c|c|c|c|c|}
\hline \multirow[t]{2}{*}{ Model } & \multirow{2}{*}{$\begin{array}{l}\text { Data source for } \\
\text { setting the model } \\
\text { parameters }\end{array}$} & \multirow[t]{2}{*}{ Figure } & \multirow[t]{2}{*}{ Observer } & \multicolumn{2}{|c|}{ RMS error $(\mathrm{dB})$} \\
\hline & & & & Mon match & Bin match \\
\hline \multirow[t]{2}{*}{ Two-stage } & \multirow[t]{2}{*}{ Meese et al. (2006) } & 4 and 6 & DHB & 0.88 & 0.97 \\
\hline & & 4 & DJH & 0.98 & 0.78 \\
\hline \multirow[t]{2}{*}{ Two-stage } & \multirow[t]{2}{*}{ Experiment I } & not shown & DHB & 1.15 & 1.04 \\
\hline & & not shown & DJH & 1.04 & 0.76 \\
\hline \multirow[t]{2}{*}{ Linear summation } & \multirow[t]{2}{*}{$\mathrm{n} / \mathrm{a}$} & 6 and 7 & DHB & 2.54 & 3.52 \\
\hline & & not shown & DJH & 2.76 & 3.22 \\
\hline \multirow[t]{2}{*}{ Quadratic summation } & \multirow[t]{2}{*}{$\mathrm{n} / \mathrm{a}$} & 6 and 7 & DHB & 1.09 & 1.69 \\
\hline & & not shown & DJH & 1.42 & 1.33 \\
\hline
\end{tabular}

The top two rows are for predictions of the two-stage model using the parameter sets from the earlier fits to contrast discrimination data from Meese et al. (2006) and Experiment I (see Table 1). The bottom two rows are for predictions of the linear summation and quadratic summation models.

before (top half of Table 1), and solving the model equation, $r e s p_{\text {standard }}=r e s p_{\text {test }}$ for test contrast. With zero degrees of freedom, the model predictions are very good (see Table 2 for RMS errors). The model correctly predicts that at low levels of 'right' eye standard contrast $(-10 \mathrm{~dB}$; open circles), matching contrast depends on the 'left' eye standard contrast (abscissa). However, as the 'right' eye standard contrast increases, perception becomes dominated by this contrast until at $30 \mathrm{~dB}$ (filled squares), it is essentially independent of 'left' eye standard contrast (abscissa).

Results for the monocular and binocular matches are remarkably similar, as might be guessed from the well-known observation that the contrast of the world does not seem to change much when you close one eye. However, there are some detailed differences in both data and model, which are most apparent around the diagonal lines in Fig. 4. (These lines indicate the contrasts for which the 'left' eye contrast is equal in the two stimuli.) In the monocular case, the data and model predictions approach this line, whereas in the binocular case, both data and model fall below it. As the monocular and binocular tests were matched to exactly the same standards, the implication is that the binocular contrasts appeared somewhat higher than monocular contrasts in our experiment. The model predicts this difference, as shown by the direct comparison of its monocular and binocular contrast responses in Fig. 5. The results of Legge and Rubin's (1981) contrast-matching experiment are also consistent with this conclusion. Note, however, that the monocular condition in our experiment (and Legge and Rubin's) is not equivalent to closing one eye; in our case contrast goes to zero in one eye but mean luminance does not.

Binocular matches to standards in which both eyes were presented with the same contrasts do fall directly on the diagonal lines, indicating a veridical match that helps to validate our methods (see the four symbols for which this is so in each of panels C and D in Fig. 4). 

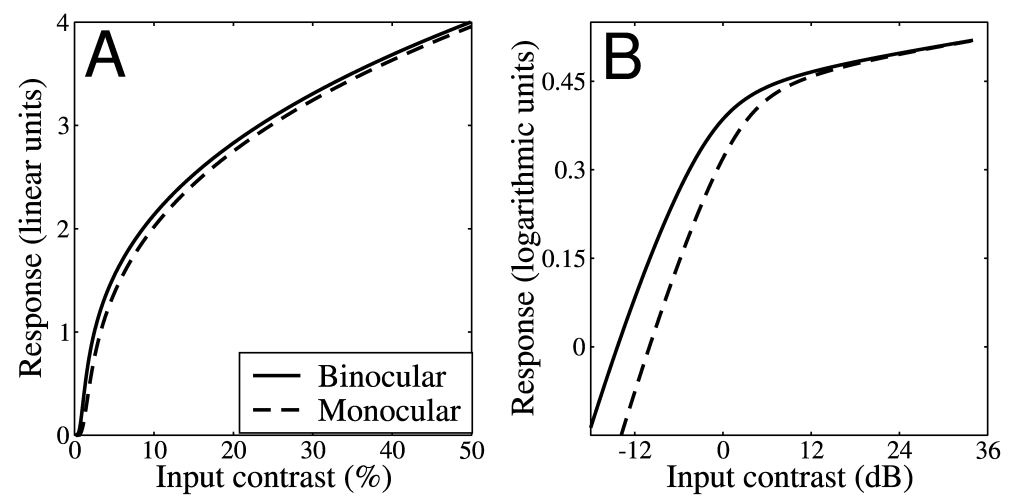

Figure 5. Two-stage model response to binocular (solid) and monocular (dashed) stimulation on (a) double linear and (b) double log axes. Note that in (a) the two functions remain apart over the full range. On log axes (b) it is clear that the marked difference in the functions occurs at and below a contrast of $1 \%(0 \mathrm{~dB})$, barely noticeable in (a). (Model parameters are from DJH - Table 1, 2nd row). These small differences between the monocular and binocular responses are responsible for the differences between the model predictions in the upper and lower panels of Fig. 4.

\section{DISCUSSION}

Although the stimuli in our discrimination (Experiment I) and matching (Experiment II) experiments were very similar, the two tasks tap different aspects of the two-stage model. In the discrimination task, performance is dependent on the output of the entire processing sequence - all six model parameters are important. But for the matching task, if the responses to the two stimuli at the output of the binocular summation box are equal, then it follows that mean responses must also be equal at the final output of the model. Therefore, model behaviour in the matching task is controlled by only two parameters: the initial exponent $m$, and the first stage saturation constant $S$. Nevertheless, the model's success is not trivial, as we now show by comparing it with two other parameter-free models (Legge, 1984b; Legge and Rubin, 1981). In the 'linear summation model', the contrasts in the two eyes are summed giving: resp $=L+R$. (For the matching task considered here, this is equivalent to an averaging model in which resp $=(L+R) / 2$, because the denominator of 2 appears on both sides of the matching equation.) In the "quadratic summation model', signals are squared before summing giving: resp $=L^{2}+R^{2}$. Neither of these simple models can handle the complexities of contrast discrimination data here or elsewhere (e.g. Legge and Foley, 1980), even when further processing is performed after binocular summation (Meese et al., 2006). Nevertheless, we ask how they might compare with the two-stage model on the less challenging task of contrast-matching. Figure 6 shows that the linear and quadratic models capture the general form of the data quite well, but fail to capture the details. For example, both models underestimate the binocular contrasts needed to achieve a match when the 'right' eye contrast is high (filled squares in Figs 6(c) and 6(d)). The linear 


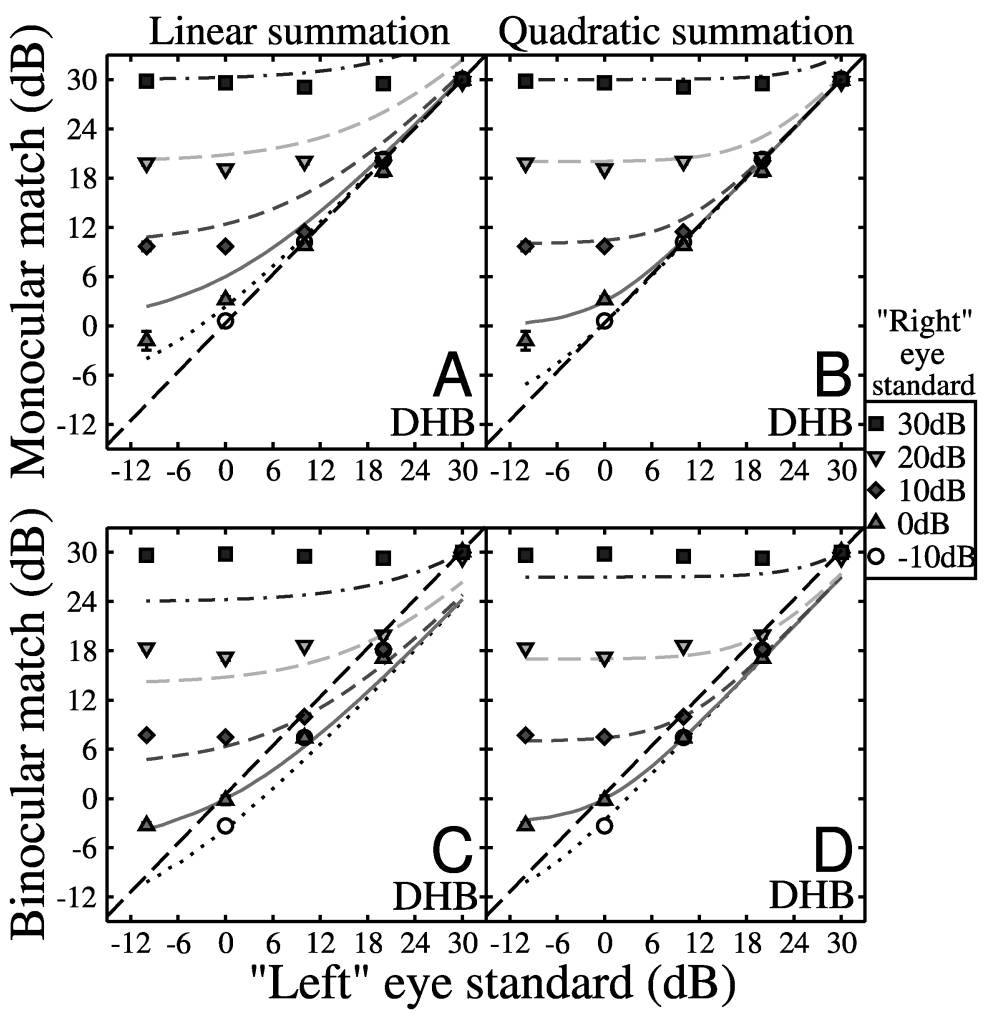

Figure 6. Predictions of the linear summation model (left) and quadratic summation model (right). Data are for DHB, replotted from Fig. 4 and duplicated in left and right panels. Axes and symbols are as Fig. 4.

model also overestimates the monocular contrasts needed to make the match when the 'right' eye contrast is low (upwards triangles in Fig. 6(a)).

By plotting the results on linear axes (Fig. 7) a further comparison is available between all three models. This method emphasizes the linear matching functions for the linear summation model (Figs 7(a) and 7(d)), which are clearly inconsistent with the data. This observation is valuable because one canonical model of contrast discrimination (pedestal masking) supposes a linear transducer, with facilitation explained by uncertainty (Pelli, 1985), and masking explained by multiplicative noise (Burton, 1981; Legge et al., 1987). We cannot rule this out, but we do note that such a model would be limited to describing discrimination (and detection) performance, and could not describe the contrast-matching results here without modification. This is because neither uncertainty nor multiplicative noise affects the matching of mean responses, and so this model reduces to the failed linear transducer of Figs 7(a) and 7(d).

Figure 7 also emphasizes the failings of the quadratic summation model at the highest 'right' eye contrast (solid squares) in Fig. 7(b). By comparison, the deviations between model and data for the two-stage model are less severe. Finally, 

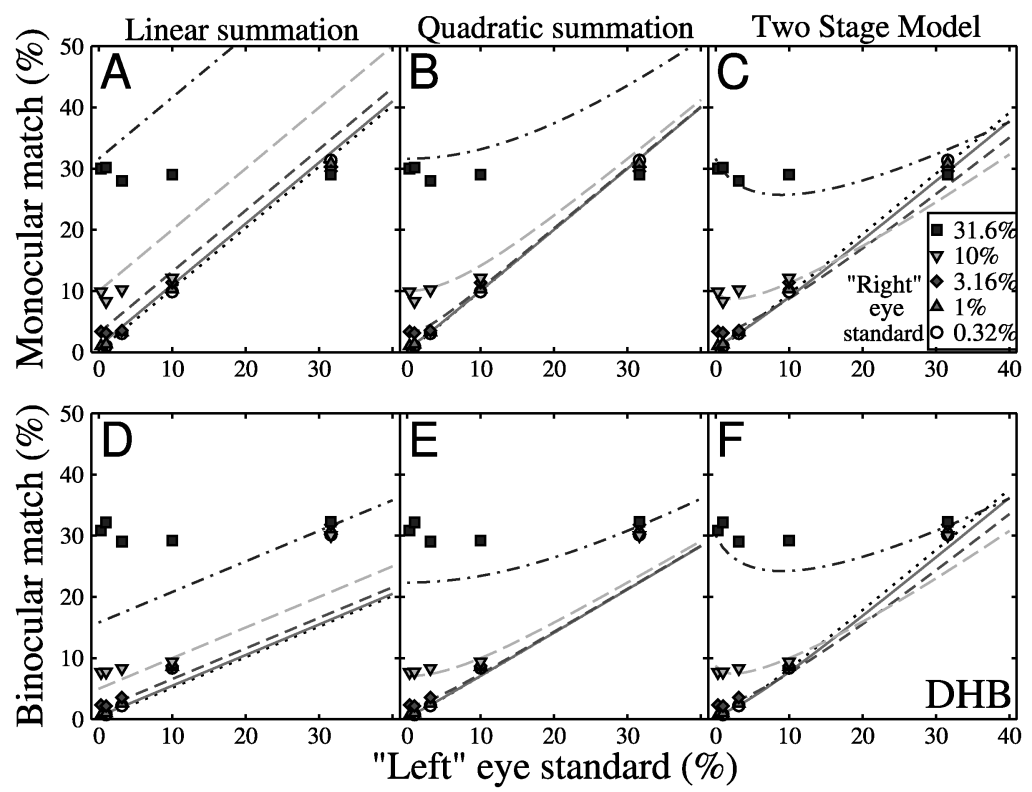

Figure 7. Matching data for subject DHB and predictions of three models (different columns), replotted on linear axes. Other details are as for Figs 4 and 5.

note that both the linear and quadratic summation models predict markedly different matching functions for the monocular and binocular conditions (compare top and bottom rows in Fig. 7). However, both the data and the two-stage model show minor differences only.

A formal comparison between the quality of fits (on the log-axes of Figs 4 and 5) is shown in Fig. 8 for both observers. The best performance by the linear model is not as good as the worst performance by the quadratic model. Similarly (though the differences are much less), the best performance by the quadratic model is not as good as the worst performance by the two-stage model. The inadequacy of the linear summation (binocular averaging) model has been noted before (Legge and Rubin, 1981; Meese et al., 2006). Meese et al. (2006) also preferred the two-stage model to the quadratic summation model. The results here confirm those conclusions.

\section{Twin-summation model}

Another model considered by Meese et al. (2006) was the so-called twin summation model - a generalization of the model proposed by Maehara and Goryo (2005). In that model, binocular summation occurs in both an excitatory stream and in a parallel suppressive stream. Consequently, the suppressive stream affects the excitatory stream after the signals have been combined across eyes. Although different in formal expression, the twin summation model behaved in a very similar way to the two-stage model in all of the tests performed by Meese et al. (2006). For brevity, we have not shown the twin-summation model here, but again we found 


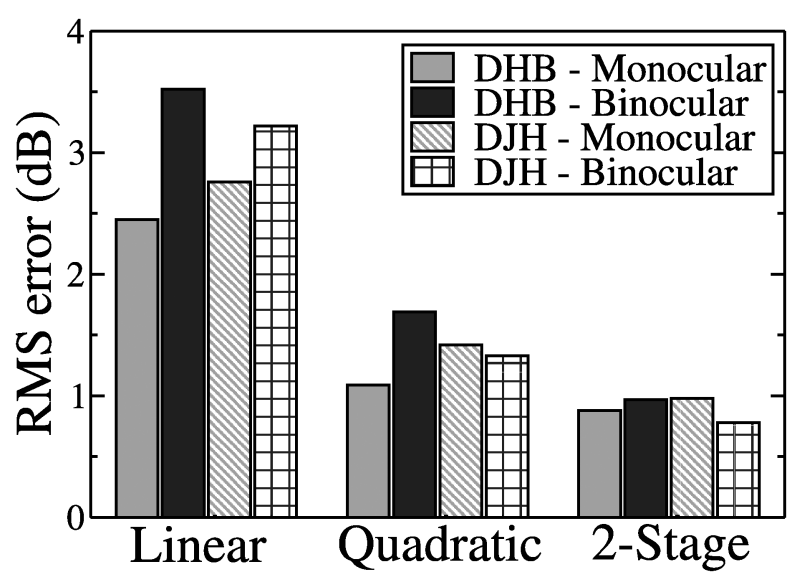

Figure 8. RMS errors $(\mathrm{dB})$ for three different models. In all cases, the model predictions were generated with no free parameters.

that it behaved very much like the two-stage model. This is not surprising, as it produces a response surface very similar to that in Fig. 1 (not shown). We are presently looking towards experiments involving cross-orientation suppression to tease these two models apart (Baker et al., 2007; Meese and Hess, 2004).

\section{Weighted averaging and adding: the best of both worlds}

The binocular visual system faces two competing demands. On the one hand, when detecting weak signals it is advantageous to sum those signals across the eyes to improve the signal-to-noise ratio. On the other hand, in suprathreshold environments, a mandatory summing or averaging of binocular signals would be very confusing, because the response would be halved when one eye is closed. In this situation, some form of normalization is required to achieve 'ocularity invariance' (the same response for one and two eyes). It would seem that the visual system has evolved to meet both of these requirements and our model suggests a framework in which this is achieved. At very low contrasts, the denominator of stage 1 is dominated by the saturation constant $(S)$, meaning that suppressive effects are weak. Consequently, the visual system benefits from the almost linear combination of the signal contrasts in the two eyes. However, at higher contrasts, the benefit due to summing is offset by almost complementary effects of suppression. The consequence is that neither contrast discrimination nor contrast-matching change very much when the signal and pedestal are added or removed from one eye.

Another way of thinking about the suprathreshold process is to see it as weighted averaging ( $c f$. Hering's law of complementary shares; de Weert and Levelt, 1974), where the relative weights are a function of relative contrast between the eyes. Thus, the initial binocular response binsum equation (1) can be re-expressed as:

$$
\text { binsum }=w_{L} L^{m-1}+w_{R} R^{m-1},
$$


where $w_{L}=L /(S+L+R), w_{R}=R /(S+L+R)$. The constant $S$ is small $(<1 \%)$ and so at suprathreshold contrasts the weights equal the relative contrasts seen by each eye: $w_{L} \approx L /(L+R), w_{R} \approx R /(L+R)$. If both eyes see the same contrast, then each eye has a weight of 0.5 and averaging occurs (along with contrast compression, with exponent $m-1 \approx 0.3$ ). But when there is contrast in one eye only, that eye receives a weight of 1 and the other eye, a weight of zero. The response to one eye is thus (almost) the same as for two eyes, except at low contrasts where $S$ plays an important role (Fig. 5(b)). The two-stage model clearly implements a form of Hering's Law at suprathreshold contrasts - each eye is favoured in proportion to the strength of its input.

Finally, a striking parallel can be drawn between the situations of binocular summation and spatial summation of contrast. In the spatial case, it is advantageous to pool over large areas when detecting weak signals, but in suprathreshold conditions, one does not want perceived contrast to change much with an increase in stimulus size. Meese et al. (2005) suggested that spatial pooling might be more extensive than is often supposed, but hidden at moderate to high contrasts by a complementary process of suppression, just like the binocular case considered here.

\section{SUMMARY AND CONCLUSIONS}

Experiment I was a novel contrast discrimination experiment in which mask and pedestal gratings were presented to different eyes over a range of contrasts for each. When mask contrast was low, thresholds (in the pedestal eye) were facilitated when the pedestal contrast was also low, but masking occurred at higher pedestal contrasts. As mask contrast increased (in the eye without the signal), the masking functions were elevated, but the region of facilitation shifted to higher pedestal contrasts (i.e. the dip of the pedestal masking function shifted to the right). These features of the data were predicted by our two-stage model of contrast gain control, whose parameters were fixed by fitting to a different data set gathered elsewhere (Meese et al., 2006). With the same fixed parameters, the model accurately predicted behaviour in a subjective (matching) task (Experiment II), in which a grating stimulus with unbalanced contrasts in the two eyes was matched to either a monocular or binocular test grating. In this task, our model outperformed both a linear summation model and a quadratic summation model. The good model performance in the two tasks here indicates that the heights and gradients of the binocular response surface in Fig. 2 are a good description of early spatial vision. In spite of the uncertainty that surrounds interpretation of the second stage of our model (see Introduction), this link between objective (performance) measures and perception (Swanson et al., 1984) is an encouraging step towards a unified account of binocular spatial vision. 


\section{Acknowledgements}

This work was supported by a grant from Engineering and Physical Sciences Research Council (GR/S74515/01) awarded to Tim Meese and Mark Georgeson.

\section{REFERENCES}

Baker, D. H., Meese, T. S. and Summers, R. J. (2007). Psychophysical evidence for two routes to suppression before binocular summation of signals in human vision, Neurosci., in press.

Burton, G. J. (1981). Contrast discrimination by the human visual system, Biological Cybernetics 40, 27-38.

Campbell, F. W. and Green, D. G. (1965). Monocular versus binocular visual acuity, Nature 208, 191-192.

de Weert, Ch. M. M. and Levelt, W. J. M. (1974). Binocular brightness combinations: additive and nonadditive aspects, Perception and Psychophysics 15, 551-562.

Ding, J. and Sperling, G. (2006). A gain-control theory of binocular combination, Proc. Nat. Acad. Sci. U.S.A. 103, 1141-1146.

Finney, D. J. (1971). Probit Analysis. Cambridge University Press, Cambridge, UK.

Foley, J. M. (1994). Human luminance pattern-vision mechanisms: masking experiments require a new model, J. Optic. Soc. Amer. A 11, 1710-1719.

Georgeson, M. A. and Meese, T. S. (2005). Binocular summation at contrast threshold: a new look, Perception 34(Suppl.), 138.

Georgeson, M. A., Meese, T. S. and Baker, D. H. (2005). Binocular summation, dichoptic masking and contrast gain control, J. Vision 5, 797.

Heeger, D. J. (1992). Normalization of cell responses in cat striate cortex, Visual Neurosci. 9, 181-197.

Holmes, D. J. and Meese, T. S. (2004). Grating and plaid masks indicate linear summation in a contrast gain pool, J. Vision 4, 1080-1089.

Kingdom, F. A. and Whittle, P. (1996). Contrast discrimination at high contrasts reveals the influence of local light adaptation on contrast processing, Vision Research 36, 817-829.

Kontsevich, L. L., Chen, C. and Tyler, C. W. (2002). Separating the effects of response nonlinearity and internal noise psychophysically, Vision Research 42, 1771-1784.

Legge, G. E. (1984a). Binocular contrast summation-I. Detection and discrimination, Vision Research 24, 373-383.

Legge, G. E. (1984b). Binocular contrast summation-II. Quadratic summation, Vision Research 24, 385-394.

Legge, G. E. and Foley, J. M. (1980). Contrast masking in human vision, J. Optic. Soc. Amer. 70, $1458-1471$.

Legge, G. E. and Rubin, G. S. (1981). Binocular interactions in suprathreshold contrast perception, Perception and Psychophysics 30, 49-61.

Legge, G. E., Kersten, D. and Burgess, A. E. (1987). Contrast discrimination in noise, J. Optic. Soc. Amer. A 4, 391-404.

Li, B., Peterson, M. R., Thompson, J. K., Duong, T. and Freeman, R. D. (2005). Cross-orientation suppression: monoptic and dichoptic mechanisms are different, J. Neurophysiol. 94, 1645-1650.

McIlhagga, W. (2004). Denoising and contrast constancy, Vision Research 44, 2659-2666.

McIlhagga, W. and Peterson, R. (2006). Sinusoid = light bar + dark bar?, Vision Research 46, 1934 1945.

Macknik, S. L. and Martinez-Conde, S. (2004). Dichoptic visual masking reveals that early binocular neurons exhibit weak interocular suppression: implications for binocular vision and visual awareness, J. Cognit. Neurosci. 16, 1049-1059. 
Maehara, G. and Goryo, K. (2005). Binocular, monocular and dichoptic pattern masking, Optical Review 12, 76-82.

Meese, T. S. and Hess, R. F. (2004). Low spatial frequencies are suppressively masked across spatial scale, orientation, field position, and eye of origin, J. Vision 4, 843-859.

Meese, T. S. and Hess, R. F. (2005). Interocular suppression is gated by interocular feature matching, Vision Research 45, 9-15.

Meese, T. S., Georgeson, M. A. and Hess, R. F. (2004). Binocular summation, interocular suppression and contrast gain control: psychophysical model and data, Perception 33 (Suppl.), 41.

Meese, T. S., Hess, R. F. and Williams, C. B. (2005). Size matters, but not for everyone: individual differences for contrast discrimination, J. Vision 5, 928-947.

Meese, T. S., Georgeson, M. A. and Baker, D. H. (2006). Binocular contrast vision at and above threshold, J. Vision 6, 1224-1243.

Mullen, K. T. and Losada, M. A. (1994). Evidence for separate pathways for color and luminance detection mechanisms, J. Optic. Soc. Amer. A 11, 3136-3151.

Nelder, J. A. and Mead, R. (1965). A simplex method for function minimization, Computer Journal 7, 308-313.

Pelli, D. G. (1985). Uncertainty explains many aspects of visual contrast detection and discrimination, J. Optic. Soc. Amer. A 2, 1508-1532.

Petrov, Y. and McKee, S. P. (2006). The effect of spatial configuration on surround suppression, $J$. Vision 6, 224-238.

Petrov, Y., Verghese, P. and McKee, S. P. (2006). Collinear facilitation is largely uncertainty reduction, J. Vision 6, 170-178.

Ross, J. and Speed, H. D. (1991). Contrast adaptation and contrast masking in human vision, Proc. Roy. Soc. London B 246, 61-69.

Ross, J., Speed, H. D. and Morgan, M. J. (1993). The effects of adaptation and masking on incremental thresholds for contrast, Vision Research 33, 2051-2056.

Sengpiel, F. and Vorobyov, V. (2005). Intracortical origins of interocular suppression in the visual cortex, J. Neurosci. 25, 6394-6400.

Swanson, W. H., Wilson, H. and Giese, S. C. (1984). Contrast matching data predicted from contrast increment thresholds, Vision Research 24, 63-75.

Truchard, A. M., Ohzawa, I. and Freeman, R. D. (2000). Contrats gain control in the visual cortex: Monocular versus binocular mechanisms, J. Neurosci. 20, 3017-3032.

Walker, G. A., Ohzawa, I. and Freeman, R. D. (1998). Binocular cross-orientation suppression in the cat's striate cortex, J. Neurophysiol. 79, 227-239. 